\title{
Comparison of anterior decompression and fusion versus laminoplasty in the treatment of multilevel cervical ossification of the posterior longitudinal ligament: a systematic review and meta-analysis
}

\author{
This article was published in the following Dove Press journal: \\ Therapeutics and Clinical Risk Management \\ 26 April 2016 \\ Number of times this article has been viewed
}

\author{
Weijun Liu',* \\ Ling $\mathrm{Hu}^{2, *}$ \\ Po-Hsin $\mathrm{Chou}^{3}$ \\ Ming Liu' \\ Wusheng Kan' \\ Junwen Wang' \\ 'Department of Orthopedics, Pu Ai \\ Hospital, Affiliated to Tongji Medical \\ College, Huazhong University of \\ Science and Technology, Wuhan, \\ People's Republic of China; \\ ${ }^{2}$ Department of Anesthesiology, \\ Tianyou Hospital, Affiliated to Wuhan \\ University of Science and Technology, \\ Wuhan, People's Republic of China; \\ ${ }^{3}$ Department of Orthopedics \& \\ Traumatology, Taipei Veterans \\ General Hospital, School of Medicine, \\ National Yang-Ming University, Taipei, \\ Taiwan, ROC \\ *These authors contributed equally \\ to this work
}

Purpose: A meta-analysis was conducted to evaluate the clinical outcomes, complications, reoperation rates, and late neurological deterioration between anterior decompression and fusion (ADF) and laminoplasty (LAMP) in the treatment of multilevel cervical ossification of the posterior longitudinal ligament (OPLL).

Methods: All related studies published up to August 2015 were acquired by searching PubMed and EMBASE. Exclusion criteria were case reports, revision surgeries, combined anterior and posterior surgeries, the other posterior approaches including laminectomy or laminectomy and instrumented fusion, non-English studies, and studies with quality assessment scores of $<7$. The main end points including Japanese Orthopedic Association (JOA) score, recovery rate of JOA, cervical lordosis, complication rate, reoperation rate, and late neurological deterioration were analyzed. All available data was analyzed using RevMan 5.2.0 and Stata 12.0.

Results: A total of seven studies were included in the meta-analysis. The mean surgical level of ADF was 3.1, and the mean preoperative occupation ratios of ADF and LAMP group were $55.9 \%$ and $51.9 \%$, respectively. No statistical difference was observed with regard to preoperative occupation ratio and preoperative JOA score. Although LAMP group had a higher preoperative cervical lordosis than ADF group $(P<0.05$, weighted mean difference [WMD] $=-5.73,95 \%$ confidence interval $[\mathrm{CI}]=-9.67--1.80)$, significantly decreased cervical lordosis was observed in LAMP group after operation. ADF group had higher postoperative JOA score $(P<0.05$, $\mathrm{WMD}=2.18,95 \% \mathrm{CI}=0.98-3.38)$ and neurological recovery rate $(P<0.05, \mathrm{WMD}=27.22,95 \%$ $\mathrm{CI}=15.20-39.23)$. Furthermore, $\mathrm{ADF}$ group had a lower late neurological deterioration rate than the LAMP group $(P<0.05$, risk difference $=0.16,95 \% \mathrm{CI}=0.04-0.73)$. The complication rates of both groups had no statistical difference. However, LAMP group had a significantly lower reoperation rate than $\mathrm{ADF}$ group. The reoperation rate of ADF group (20.5\%) was almost six times that of LAMP group (3.5\%).

Conclusion: Our meta-analysis suggested that ADF was associated with better postoperative neurological function, neurological recovery rate, and less late neurological deterioration than LAMP in the treatment of multilevel cervical OPLL with a high mean occupation ratio. LAMP was associated with a decreased postoperative cervical lordosis, which might be a cause of late neurological deterioration. The complication rates of both groups showed no statistical difference. However, the reoperation rate was significantly higher in ADF group compared with LAMP group. Benefits and risks should be balanced when ADF or LAMP is selected.

Keywords: anterior decompression and fusion, laminoplasty, ossification of the posterior longitudinal ligament, late neurological deterioration, meta-analysis 


\section{Introduction}

Ossification of the posterior longitudinal ligament (OPLL) is an important cause of cervical myelopathy. The incidence of OPLL ranges from $1.9 \%$ to $4.3 \%$ in East Asian countries and from $0.01 \%$ to $1.7 \%$ in Caucasian populations. ${ }^{1,2}$ Although many clinical features of cervical OPLL are similar to those of cervical myelopathy caused by cervical disc herniation, the former still has several unique characteristics. Conservative treatment is usually ineffective for moderate-to-severe myelopathy caused by OPLL; instead, surgical treatment is the first option in these cases.

There are two representative surgical approaches: anterior decompression and fusion (ADF) and laminoplasty (LAMP). However, some controversies still remain on surgical selection. Removal of OPLL from anterior approach seems to be radical to decompress the spinal cord, and fusion can establish a cervical stability that is conducive to relieve pressure on the levels of compressed cervical cord. ${ }^{3}$ Meanwhile, complications and technical problems, including dural tear, graft extrusion, insufficient decompression, and so on, are still great concerns, especially when multiple levels and high occupation ratios are involved. ${ }^{4,5}$ ADF remains a significant surgical technical challenge.

The indirect decompression via LAMP and cervical lordosis alignment allows the spinal cord to float away from ventral compression. However, if posterior shift of the cord is insufficient, ventral constriction of the cord may persist, leading to diminished recovery of neurological function. Admittedly, the surgical technique is less difficult for LAMP than ADF.

At present, no standards or guidelines exist for the treatment of OPLL. We performed this meta-analysis to evaluate the clinical outcomes, complications, reoperation rates, and late neurological deterioration between ADF and LAMP in the treatment of cervical OPLL with the aim of trying to find the evidences for how to balance the benefits and risks of the aforementioned surgical approaches.

\section{Methods}

We developed a protocol prior to this systematic review, which was registered in PROSPERO. The registration number is CRD42015025032.

\section{Search strategy}

The primary sources of the studies reviewed in this metaanalysis were PubMed and EMBASE. The search included literature exclusively in English and published up to August 2, 2015. The following terms were used in our search: anterior AND (ossification of the posterior longitudinal ligament OR ossified posterior longitudinal ligament OR calcification of the posterior longitudinal ligament). Reference lists of all included studies were scanned to identify potentially relevant studies. Two reviewers independently screened the titles and abstracts of studies identified from the search. Full-text copies of all potentially relevant studies were obtained.

\section{Inclusion and exclusion criteria}

Studies were included if they met the following criteria: 1) study design: prospective or retrospective comparative study; 2) patients with cervical myelopathy due to OPLL, excluding patients with tumors, trauma, infection, previous surgeries, revision surgeries, combined anterior and posterior surgeries, and other posterior approaches including laminectomy or laminectomy and instrumented fusion; 3) purpose of the studies: to compare clinical outcome differences between ADF and LAMP; 4) outcome measurements: including Japanese Orthopedic Association (JOA) score, neurological recovery rate, cervical lordosis, complications, reoperation rate, and late neurological deterioration; and 5) published in English. Studies that did not meet these criteria were excluded.

\section{Data extraction}

The following information was extracted from each study: 1) study ID, 2) study design, 3) study location, 4) number of cases, 5) length of follow-up, 6) number of surgical levels, 7) preoperative occupation ratios, 8) space available for spinal cord, 9) operation time, 10) blood loss, 11) preoperative and postoperative JOA scores, 12) cervical lordosis, 13) neurological recovery rate, 14) complications, 15) reoperations, 16) late neurological deterioration, and 17) patient's age.

\section{Data analysis}

Statistical analysis was conducted using the Review Manager software (RevMan Version 5.2. The Nordic Cochrane Center, The Cochrane Collaboration, Copenhagen, Denmark). Heterogeneity was tested using the chi-square test and quantified by calculating the $I^{2}$ statistic, in which $P<0.05$ and $I^{2}>50 \%$ were considered statistically significant. For the pooled effects, weighted mean difference (WMD) was calculated for continuous variables according to the consistency of measurement units, and odds ratio (OR) was 
calculated for dichotomous variables. Continuous variables are presented as mean differences and $95 \%$ confidence intervals (CIs), whereas dichotomous variables are presented as ORs and 95\% CI. Random-effects or fixed-effects models were used depending on the heterogeneity of the studies included. Publication bias was tested using a funnel plot.

\section{Results}

\section{Characteristics of studies}

A total of 951 papers were first identified by screening the titles and abstracts. Of these, 935 papers were excluded because they were duplicates, irrelevant studies, case reports, revision surgeries, combined anterior and posterior surgeries, non-English studies, and reviews. The remaining 16 papers underwent a detailed and comprehensive evaluation (Figure 1). Papers with quality assessment scores of $<7$ and all other posterior approaches including laminectomy or laminectomy and instrumented fusion were excluded. The remaining seven studies were finally included in this meta-analysis. ${ }^{6-12}$ Details of these studies are listed in Table 1.

\section{Quality assessment}

Two investigators evaluated each study and extracted data independently; any disagreements were resolved by discussion. The baseline characteristics of the participants in these studies were similar. Newcastle-Ottawa quality assessment scale was used to assess the quality of the included studies. Among these studies, five of them scored 8 points and two scored 7 points. These scores indicate that the studies included in this meta-analysis were of high quality (Table 1).

\section{Surgical level and surgical approach selection}

All included papers reported the surgical levels of ADF. The mean surgical level of ADF was 3.1. The surgical levels of LAMP are shown in Table 1. Only one study selected surgical approach according to different given time of surgery (ADF group in 1997, 1999, 2001, 2003, and 2004 and LAMP group in 1996, 1998, 2000, and 2002), ${ }^{8}$ while the other six studies had no clear or definitive selection criterion for each surgical approach.

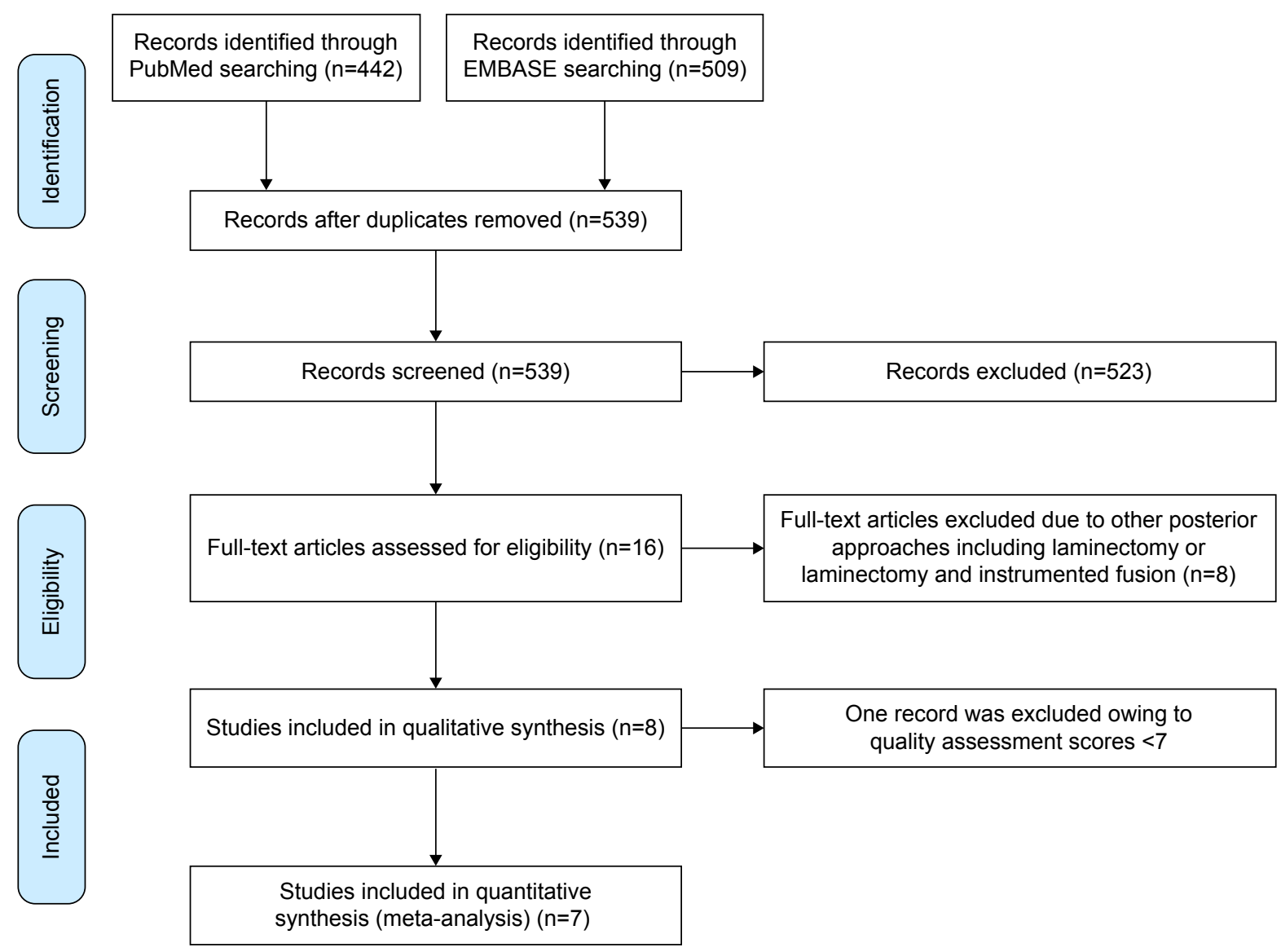

Figure I Flowchart of study selection. 


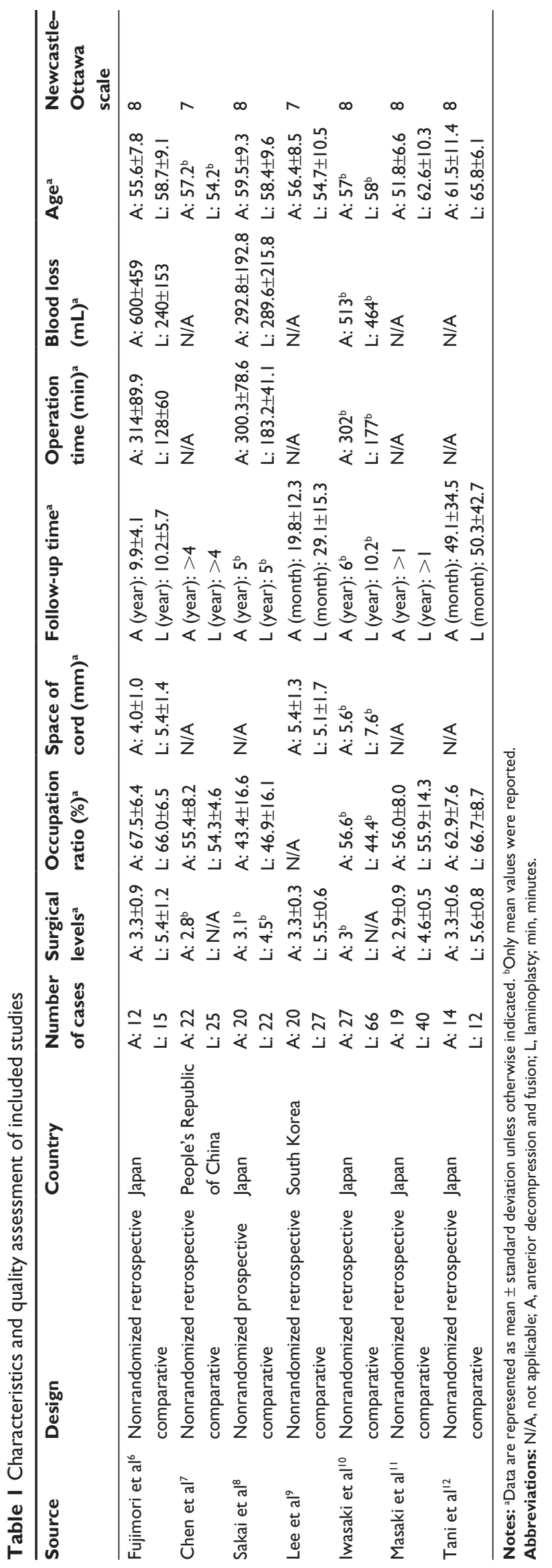

\section{Preoperative occupation ratios}

The preoperative occupation ratios were reported in six studies. Approximately 294 cases were involved, including 114 cases of ADF and 180 cases of LAMP. The mean preoperative occupation ratios were $55.9 \%$ in ADF group and $51.9 \%$ in LAMP group. There was no significant difference in the preoperative occupation ratio between $\mathrm{ADF}$ group and LAMP group $(P=0.474)$.

\section{Preoperative and postoperative JOA scores}

Preoperative and postoperative JOA scores (final follow-up) were analyzed in six studies. Standard deviation of one study was not reported; thus, the statistical data for this one were unavailable. Five studies and 201 cases were involved, including 87 cases of ADF and 114 cases of LAMP. There was no significant difference in the preoperative JOA score between ADF group and LAMP group $(P>0.05, \mathrm{WMD}=0.54$ $[-0.05,1.12]$; Figure 2$)$, and the chi-square test indicated no statistical evidence of heterogeneity $(I=0 \%, P=0.07)$. The ADF group had a significant higher postoperative JOA score than the LAMP group $(P<0.05, \mathrm{WMD}=2.18[0.98,3.38]$; Figure 2), and moderate heterogeneity existed between these studies $\left(I^{2}=76 \%, P=0.0004\right)$.

\section{Neurological recovery rate}

The recovery rates of JOA at final follow-up were analyzed in six studies. Standard deviation of one study was not reported, and the statistical data were unavailable and thus excluded. Five studies and 201 cases were involved, including 87 cases of ADF and 114 cases of LAMP. The mean recovery rate of ADF and LAMP groups was $63.9 \%$ and $40.0 \%$, respectively. The ADF group had a significantly higher postoperative neurological recovery rate than LAMP group $(P<0.05$, $\mathrm{WMD}=27.22$ [15.20, 39.23]; Figure 2). Moderate heterogeneity existed between these studies $(I=68 \%, P<0.00001)$.

\section{Preoperative and postoperative cervical lordosis}

Preoperative and postoperative cervical lordosis (final follow-up) were analyzed in three studies. A total of 116 cases were involved, including 52 cases of ADF and 64 cases of LAMP. The LAMP group had a significantly higher preoperative cervical lordosis than the ADF group $(P<0.05, \mathrm{WMD}=-5.73[-9.67,-1.80]$; Figure 3$)$, and mild heterogeneity existed between these studies $\left(I^{2}=10 \%\right.$, $P=0.004)$. There was no significant difference in the postoperative cervical lordosis between ADF group and LAMP 
2.1 Preoperative JOA score

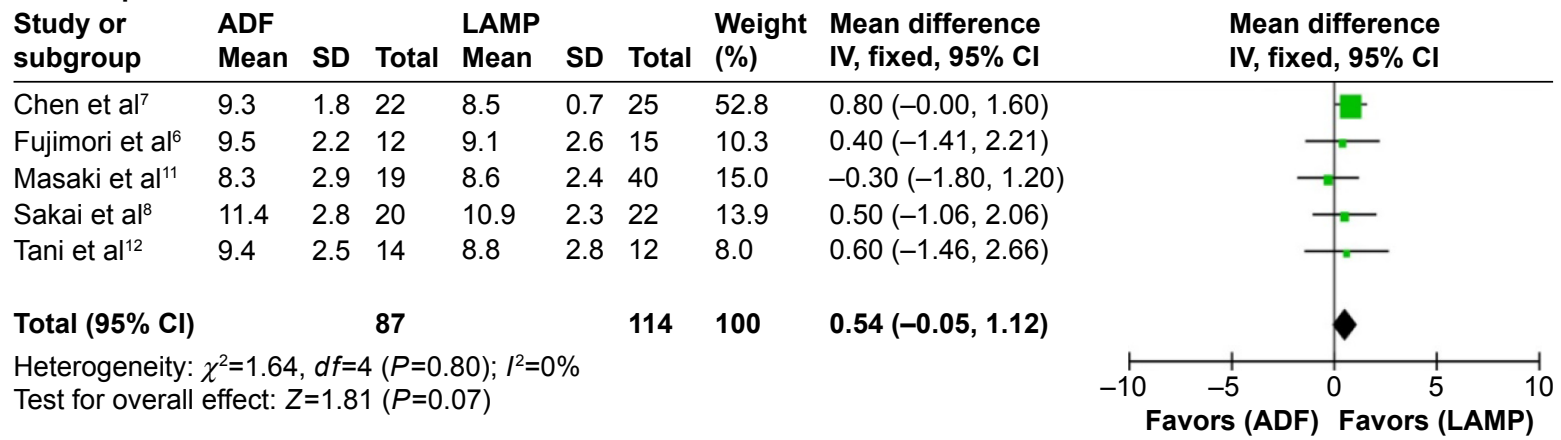

2.2 Preoperative JOA score

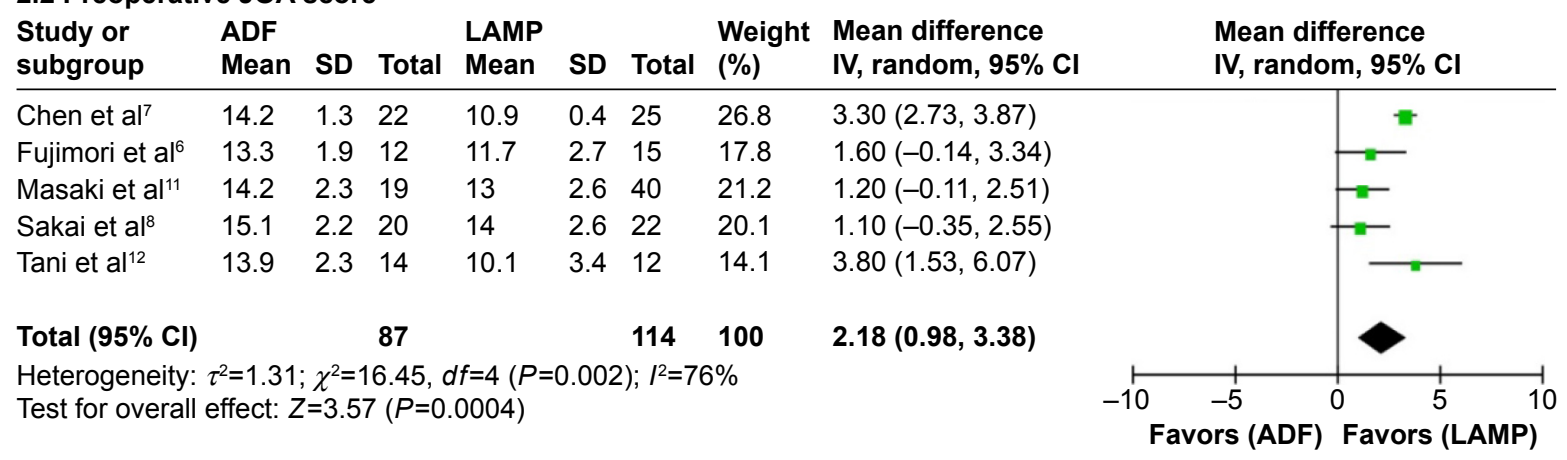

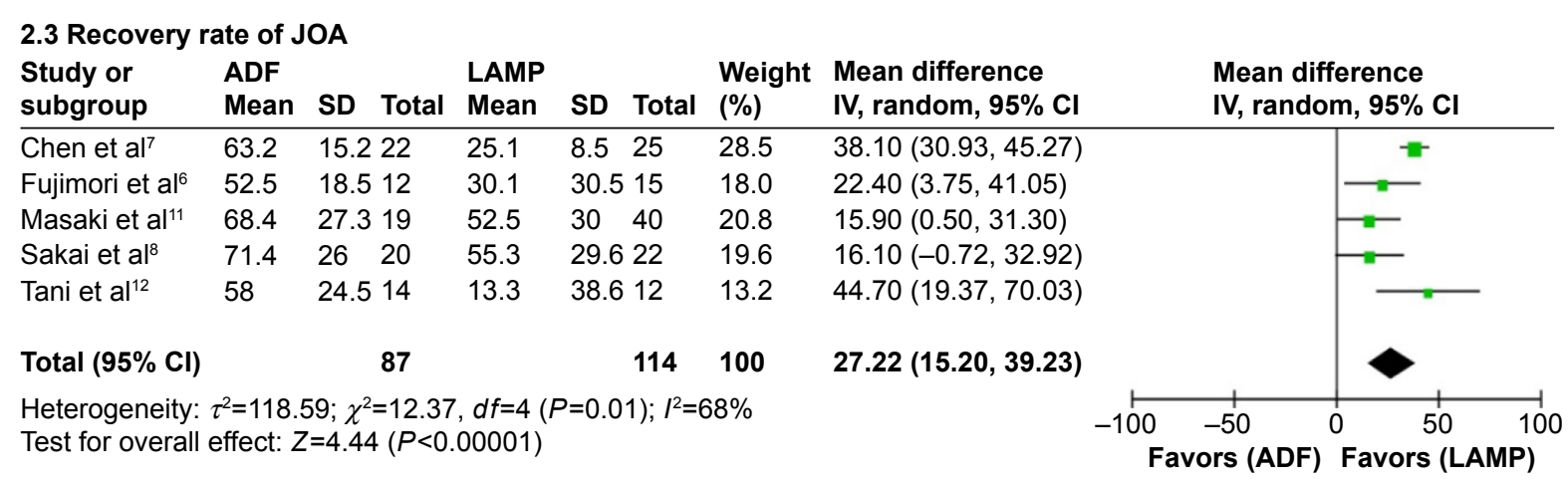

Figure 2 Forest plot illustrating the comparison in preoperative JOA score (fixed-effects model, 2.I), the postoperative JOA score (random-effects model, 2.2), and recovery rate of JOA (random-effects model, 2.3) between ADF and LAMP groups.

Abbreviations: $\mathrm{Cl}$, confidence interval; df, degrees of freedom; IV, independent variable; SD, standard deviation; JOA, Japanese Orthopedic Association; ADF, anterior decompression and fusion; LAMP, laminoplasty.

group $(P>0.05, \mathrm{WMD}=2.05[-6.23,10.34]$; Figure 3$)$, and moderate heterogeneity existed between these studies $\left(I^{2}=81 \%, P=0.63\right)$. The postoperative cervical lordosis was significantly lower than preoperative cervical lordosis in LAMP group $(P<0.05, \mathrm{WMD}=-4.05[-7.80,-0.30])$. There was no significant difference between preoperative and postoperative cervical lordosis in $\mathrm{ADF}$ group $(P>0.05$, $\mathrm{WMD}=3.09[-0.67,6.84])$.

\section{Late neurological deterioration}

Late neurological deterioration of LAMP and ADF was reported in four and five studies, respectively. Four studies and 174 cases were involved, including 75 cases of ADF and 99 cases of LAMP. The LAMP group had a significantly higher incidence of late neurological deterioration than ADF group $(P<0.05$, OR $=0.16$ [0.04, 0.73]; Figure 4). The chi-square test indicated no statistical evidence of heterogeneity $\left(I^{2}=0 \%, P=0.02\right)$.

\section{Complications}

Complications of LAMP and ADF were reported in four and five studies, respectively. Four studies and 163 cases were involved, including 74 cases of ADF and 89 cases of LAMP. There was no significant difference in complication rate 
3.1 Preoperative cervical lordosis

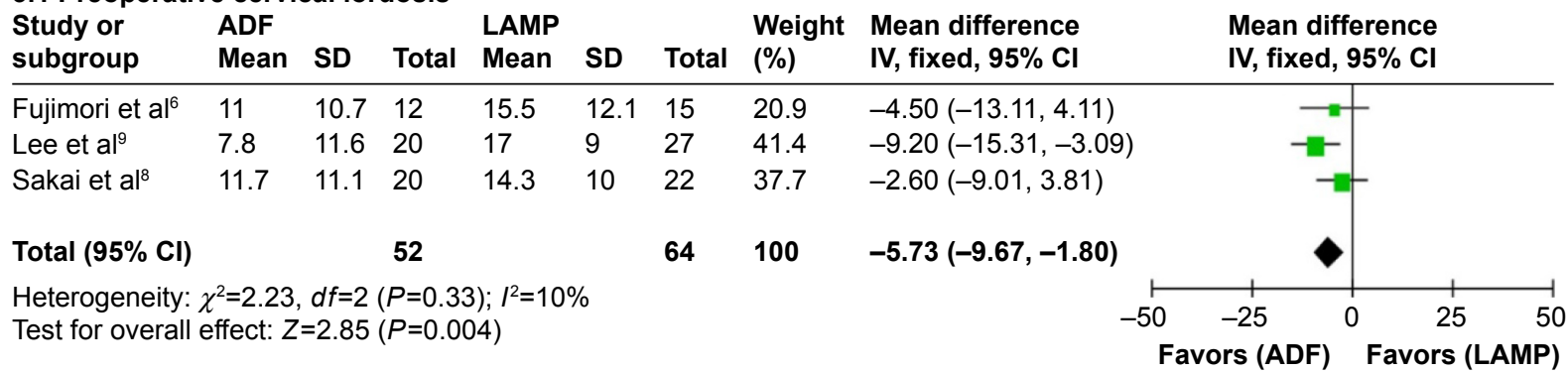

3.2 Postoperative cervical lordosis

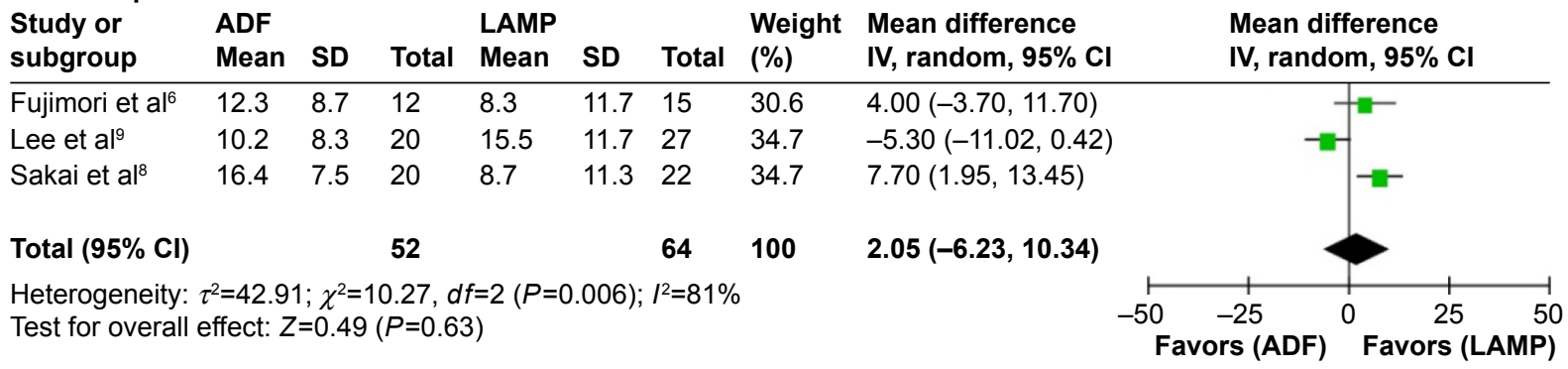

Figure 3 Forest plot illustrating the comparison in preoperative cervical lordosis (fixed-effects model, 3.I) and postoperative cervical lordosis (random-effects model, 3.2) between ADF and LAMP groups.

Abbreviations: $\mathrm{Cl}$, confidence interval; $d f$, degrees of freedom; IV, independent variable; SD, standard deviation; ADF, anterior decompression and fusion; LAMP, laminoplasty.

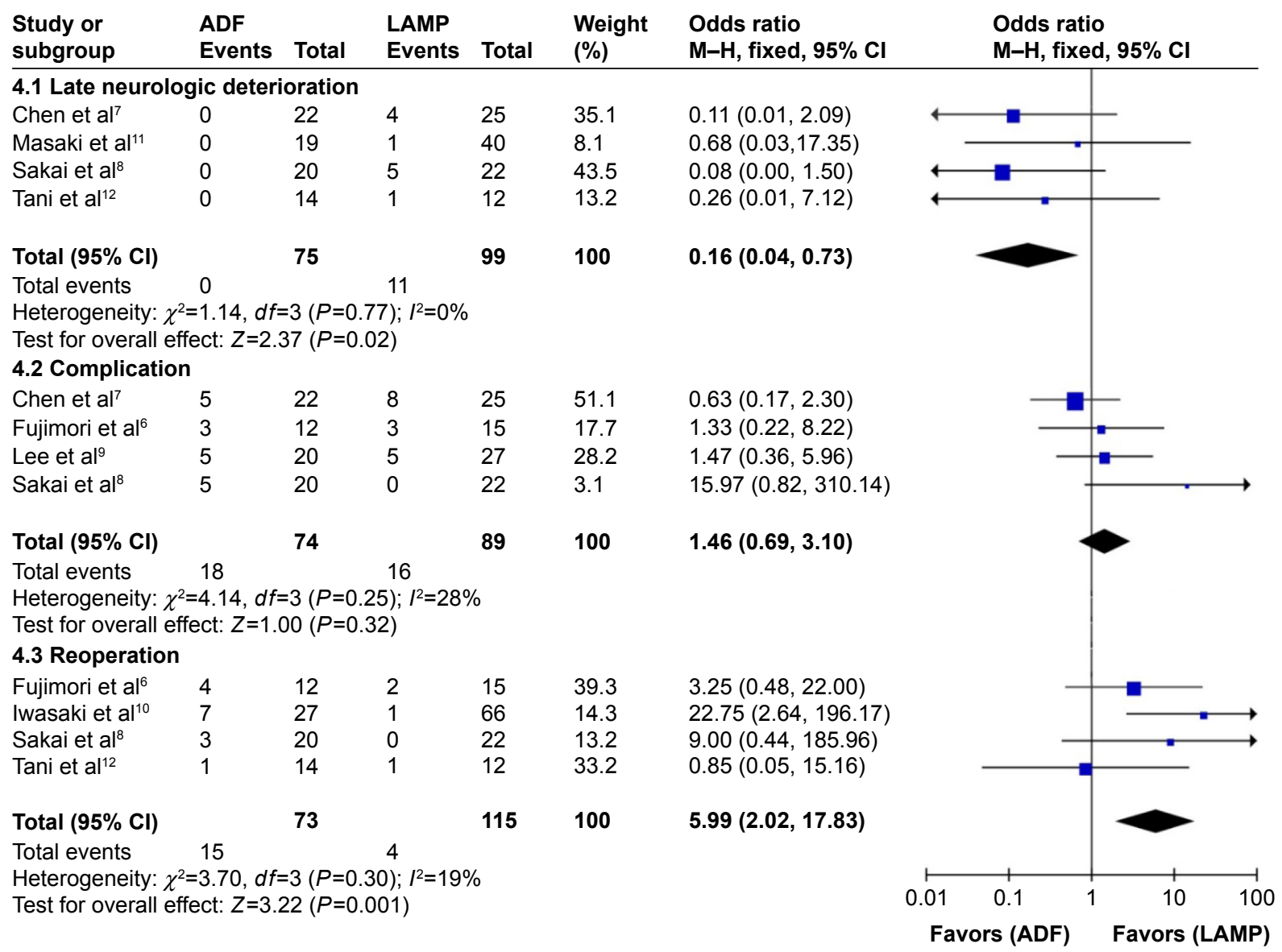

Figure 4 Forest plot (fixed-effects model) illustrating the comparison in late neurological deterioration (4.I), complication (4.2), and reoperation (4.3) between ADF and LAMP groups.

Abbreviations: $\mathrm{Cl}$, confidence interval; $d f$, degrees of freedom; M-H, Mantel-Haenszel; SD, standard deviation; ADF, anterior decompression and fusion; LAMP, laminoplasty. 
between ADF group and LAMP group $(P>0.05$, OR $=1.46$

$[0.69,3.10]$; Figure 4$)$. Mild heterogeneity existed between these studies $\left(I^{2}=28 \%, P=0.32\right)$.

\section{Reoperations}

Reoperations were reported in four studies. A total of 188 cases were involved, including 73 cases of ADF and 115 cases of LAMP. The mean reoperation rates of ADF and LAMP group were $20.5 \%$ and $3.5 \%$, respectively. The ADF group had a significantly higher reoperation rate than LAMP group $(P<0.05$, $\mathrm{OR}=5.99[2.02,17.83]$; Figure 4$)$. Mild heterogeneity existed between these studies $\left(I^{2}=19 \%, P=0.001\right)$.

\section{Publication bias}

The Stata 12.0 software (StataCorp LP, College Station, TX, USA) was used to examine the publication bias of the main results. All funnel plots were largely symmetrical including preoperative and postoperative JOA and cervical lordosis, neurological recovery rate, and complication rate (Figure 5). These results indicated that publication bias did not play a vital role in the observed effects, and the conclusions were reliable.
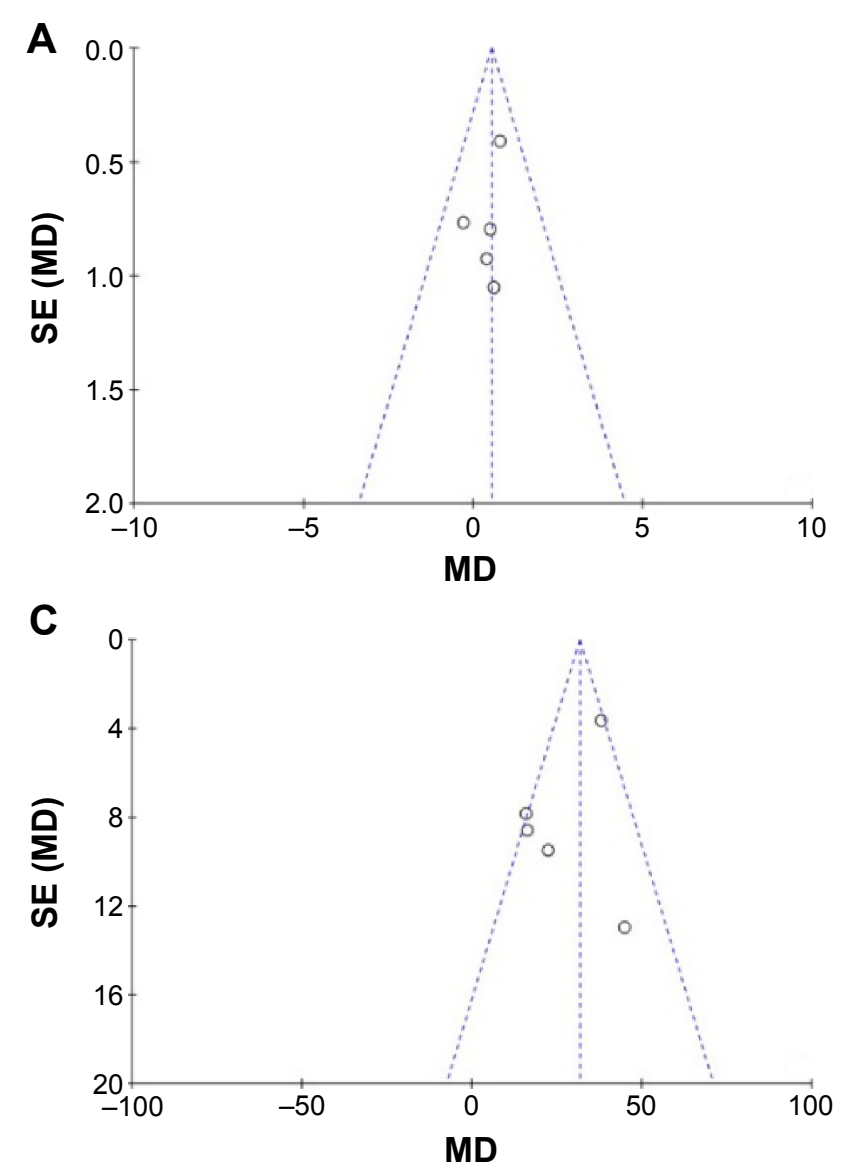

\section{Discussion}

The ideal surgical treatment option for multilevel cervical OPLL remains controversial and presents a significant surgical challenge. Previously, a systematic review that included only three papers had been published regarding decision making in the treatment of OPLL, and no definitive conclusion was reached..$^{13}$ Five meta-analysis papers about surgery approaches of cervical myelopathy have also been published, ${ }^{14-18}$ but two of them excluded the studies about OPLL, ${ }^{14,15}$ the other papers had not analyzed the OPLL separately, and the number of included studies about OPLL was equal to four or less. ${ }^{16-18}$ Moreover, the five meta-analysis papers published had conflicting results, and no consensus on neurological recovery rate had been reached. Therefore, we performed the meta-analysis to evaluate the clinical outcomes, complications, reoperations, and late neurological deterioration between ADF and LAMP in the treatment of cervical OPLL.

In our meta-analysis, seven included papers were considered to be of sufficient methodological quality. The mean surgical level of ADF was 3.1, and the mean preoperative occupation ratios of ADF and LAMP group were $55.9 \%$ and
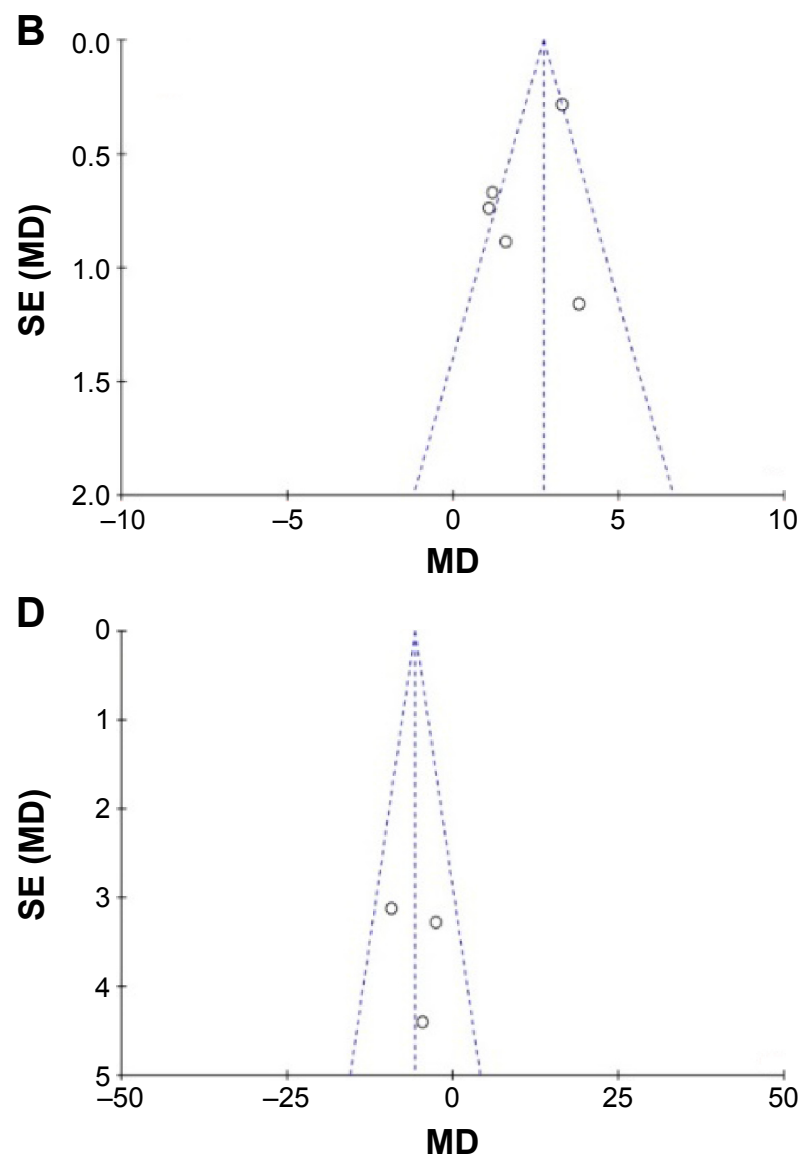

Figure 5 (Continued) 

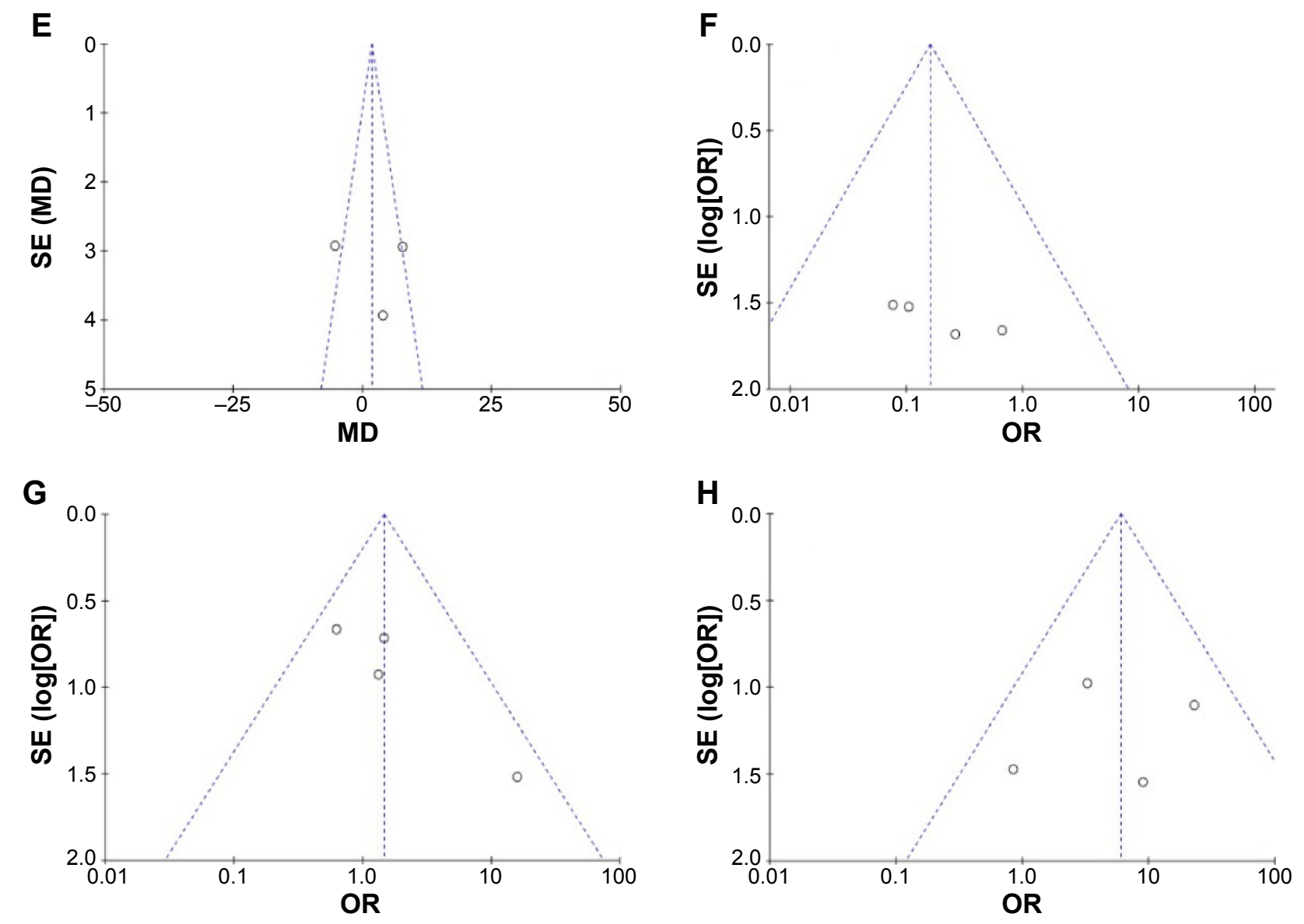

Figure 5 Funnel plot analysis of studies on preoperative JOA score (A), postoperative JOA score (B), recovery rate of JOA (C), preoperative cervical lordosis $(\mathbf{D})$, postoperative cervical lordosis $(\mathbf{E})$, late neurological deterioration $(\mathbf{F})$, complication $(\mathbf{G})$, and reoperation $(\mathbf{H})$ that shows publication bias.

Abbreviations: JOA, Japanese Orthopedic Association; MD, mean difference; OR, odds ratio; SE, standard error; RD, risk difference.

$51.9 \%$, respectively. No statistical difference was observed in preoperative occupation ratios and preoperative JOA scores. It indicated that the baseline neurological function characteristics of these two groups were similar. However, ADF group had higher postoperative JOA score and recovery rate. The mean recovery rates of ADF group and LAMP group were $63.9 \%$ and $40.0 \%$, respectively. It indicated that the direct decompression of ADF was associated with better neurological recovery and function in the treatment of cervical OPLL with a high mean occupation ratio.

LAMP relies heavily on the decompression effect for indirect decompression through a posterior shift of the spinal cord, and cervical lordosis plays an important role in preventing the static compression. Yamazaki et al ${ }^{19}$ reported that cervical lordosis of $<10^{\circ}$ or OPLL thickness of $>7 \mathrm{~mm}$ were risk factors for spinal cord contact with OPLL. Fujimori et $\mathrm{al}^{6}$ concluded that preoperative cervical lordosis of $20^{\circ}$ or more might be necessary if LAMP was applied to successfully treat OPLL with an occupation ratio of $60 \%$ or higher. The K-line was also advocated to evaluate both cervical alignment and the size of OPLL. A negative K-line slope means that the OPLL touches the spinal cord and has a significantly lower recovery rate than the positive K-line group. ${ }^{20}$ In our meta-analysis, the LAMP group had a higher preoperative cervical lordosis than ADF group.

Furthermore, postoperative kyphotic change after LAMP has also been detected. Sakai et $\mathrm{al}^{8}$ reported that it was observed in $50 \%$ of the LAMP at 5 -year follow-up. ${ }^{8}$ Biomechanical analysis of cervical OPLL indicated that stress distribution increased with the progression of kyphosis after posterior decompression, which was likely associated with the late neurological deterioration. ${ }^{21}$ In our meta-analysis, although LAMP group had a higher preoperative cervical lordosis than ADF group, cervical lordosis of LAMP group had significantly decreased after operation. The preoperative and postoperative cervical lordosis of ADF group and the postoperative cervical lordosis of both groups had no statistical difference. Meanwhile, ADF group had a lower late neurological deterioration rate than LAMP group. 
Dynamic factor can also affect clinical outcomes of LAMP. A larger postoperative $\mathrm{C} 2-\mathrm{C} 7$ range of motion was related to late neurological deterioration in patients with the segmental type of OPLL. ${ }^{22}$ The spontaneous fusion of vertebrae by the bridging of OPLL and the ossification of the anterior longitudinal ligament were considered as the most important factors protecting against deterioration in myelopathy. ${ }^{23}$ Goel et $\mathrm{al}^{24}$ found that treatment of OPLL with only fixation of the involved spinal segments without decompression had encouraging clinical outcomes. Fusion was advocated to conquer the postoperative kyphotic change and dynamic factor in the treatment of OPLL. ${ }^{5,24}$

To date, several researches that compared LAMP with laminectomy and fusion (LF) in the treatment of OPLL have been published. Chen et $\mathrm{al}^{7}$ reported that LF group had significantly higher recovery rate of JOA and postoperative JOA score than the LAMP group. ${ }^{7}$ Yuan et $\mathrm{al}^{25}$ reported that there was no statistically significant difference in the postoperative JOA scores between the two groups. Another paper did not report the outcomes of neurological function, but it did report that the LAMP group had a significantly higher incidence of progression of OPLL than the LF group. ${ }^{26}$ More studies are needed to compare LAMP and LF with regard to clinical outcomes in the treatment of OPLL and to further evaluate the impacts of fusion.

Postoperative progression of OPLL has been reported in both LAMP and ADF. Iwasaki et a ${ }^{27}$ reported that postoperative progression of OPLL after LAMP was observed in $70 \%$ of the patients over a 10 -year follow-up. Another team, Matsuoka et $\mathrm{al},{ }^{28}$ reported that a marked postoperative progression of the OPLL after ADF was observed in $16.7 \%$ of the patients over a 10 -year follow-up. Sakai et al ${ }^{8}$ reported that postoperative progression of the OPLL at 5-year follow-up period was observed in $5.0 \%$ of the ADF group and in $50.0 \%$ of the LAMP group. Progression of OPLL, loss of cervical lordosis, and dynamic factor may be the main causes of late neurological deterioration of LAMP in the treatment of OPLL. Insufficient decompression was more associated with late neurological deterioration in ADF group. ${ }^{23}$

In our meta-analysis, the mean incidence of late neurological deterioration in ADF and LAMP groups was $1.9 \%$ and $11.1 \%$, respectively. It indicated that ADF could reduce the incidence of late neurological deterioration than LAMP in the treatment of OPLL.

Nowadays, many new techniques and methods are applied to remove the OPLL and evaluate the risks in anterior approach..$^{29,30}$ No statistically significant difference was observed with regard to the complication rate of both groups in our meta-analysis. However, the reoperation rate was significantly higher in ADF group than in the LAMP group in our meta-analysis. Dural tear, graft extrusion, pseudarthrosis, and iatrogenic neurological deterioration were more often reported in ADF group, while C5 palsy and axial pain were also reported more frequently in the LAMP group. In four papers included in our analysis, the causes of reoperation were graft extrusion (6.8\%), pseudarthrosis (4.1\%), insufficient decompression (4.1\%), late neurological deterioration (2.7\%), C5 palsy (1.4\%), and hematoma (1.4\%) in ADF group (20.5\%) and hematoma (1.7\%), progression of OPLL $(0.9 \%)$, and late neurological deterioration $(0.9 \%)$ in the LAMP group (3.5\%), respectively. The reoperation rate of ADF group was almost six times that of LAMP group. Graft extrusion and pseudarthrosis were the main causes of reoperation in $\mathrm{ADF}$ group. In these studies, the mean surgical level of ADF was from 3.0 to 3.3. Multilevel cervical corpectomy was associated with increased graft-related complications compared with single-level procedure. ${ }^{31}$ Biomechanical analysis suggested that stability of long-segment anterior plate fixation after a multilevel $(\geq 2)$ corpectomy was insufficient. ${ }^{31,32}$ The cantilever force generated at the screwbone interface increased as the length of the fused segment or plate length increased. As a result, anterior plate fixation tended to fail at the caudal end of the construct in multilevel corpectomy. ${ }^{32}$ Combined anterior-posterior fixation or posterior-only fixation was recommended in multilevel cervical corpectomy, which was significantly more rigid than the anterior-only fixation. ${ }^{33,34}$ However, in the studies discussed here, only anterior fixation was performed in ADF group, which might play an important role in graft extrusion and pseudarthrosis. Insufficient decompression was another main cause of reoperation in ADF group. Continuous-type OPLL, ossification of the dura, massive bleeding from the epidural space, and technical difficulties were the possible reasons for insufficient decompression in ADF group in the treatment of cervical OPLL. ${ }^{6,10,12}$

Meanwhile, there are some limitations to this meta-analysis. First, the included publications are in English; thus, a potential language bias may exist in this meta-analysis. Second, the sample size may not be large enough to find the possible existing evidence; therefore, larger-scale and higher-quality studies are needed to provide more reliable evidence for future evaluation. Third, there was a variable length of time in the follow-ups between some of the studies, and this complicated the evaluation and comparison of the surgical results. Fourth, clinical heterogeneity may be caused by the various indications for surgery and for the use of certain surgical technologies at 
the different treatment centers. Finally, the included patients with OPLL had a high mean occupation ratio (>50\%). Some studies reported that the low occupation ratio group had significantly better clinical outcomes than high occupation ratio group $(>50 \%$ or $60 \%)$ in the treatment of cervical OPLL with LAMP; 8,10 therefore, our conclusion was based on a high mean occupation ratio. Further studies are needed regarding comparison of ADF versus LAMP in the treatment of cervical OPLL with a low mean occupation ratio.

\section{Conclusion}

In this meta-analysis, we systematically compared ADF with LAMP with regard to the clinical outcomes, complications, reoperation rates, and late neurological deterioration between ADF and LAMP in the treatment of multilevel cervical OPLL. ADF had better postoperative neurological function, neurological recovery rate, and less late neurological deterioration than LAMP in the treatment of cervical OPLL, with a high mean occupation ratio. LAMP had a decreased postoperative cervical lordosis that might be a cause of late neurological deterioration. The complication rates of both groups had no statistical difference. However, the reoperation rate was significantly higher in ADF group compared with LAMP group. The reoperation rate of ADF group was almost six times that of LAMP group. Benefits and risks should be balanced when ADF or LAMP is selected.

\section{Disclosure}

The authors report no conflicts of interest in this work.

\section{References}

1. Kalb S, Martirosyan NL, Perez-Orribo L, Kalani MY, Theodore N. Analysis of demographics, risk factors, clinical presentation, and surgical treatment modalities for the ossified posterior longitudinal ligament. Neurosurg Focus. 2011;30(3):E11.

2. Wang MY, Thambuswamy M. Ossification of the posterior longitudinal ligament in non-Asians: demographic, clinical, and radiographic findings in 43 patients. Neurosurg Focus. 2011;30(3):E4.

3. Nouri A, Tetreault L, Singh A, Karadimas SK, Fehlings MG. Degenerative cervical myelopathy: epidemiology, genetics, and pathogenesis. Spine (Phila Pa 1976). 2015;40(12):E675-E693.

4. Epstein N. The surgical management of ossification of the posterior longitudinal ligament in 51 patients. J Spinal Disord. 1993;6(5): 432-454.

5. An HS, Al-Shihabi L, Kurd M. Surgical treatment for ossification of the posterior longitudinal ligament in the cervical spine. J Am Acad Orthop Surg. 2014;22(7):420-429.

6. Fujimori T, Iwasaki M, Okuda S, et al. Long-term results of cervical myelopathy due to ossification of the posterior longitudinal alignment with an occupying ratio of $60 \%$ or more. Spine (Phila Pa 1976). 2014;39(1):58-67.

7. Chen Y, Guo Y, Lu X, et al. Surgical strategy for multilevel severe ossification of posterior longitudinal ligament in the cervical spine. J Spinal Disord Tech. 2011;24(1):24-30.
8. Sakai K, Okawa A, Takahashi M, et al. Five-year follow-up evaluation of surgical treatment for cervical myelopathy caused by ossification of the posterior longitudinal ligament: a prospective comparative study of anterior decompression and fusion with floating method versus laminoplasty. Spine (Phila Pa 1976). 2012;37(5):367-376.

9. Lee SH, Ahn Y, Lee JH. Laser-assisted anterior cervical corpectomy versus posterior laminoplasty for cervical myelopathic patients with multilevel ossification of the posterior longitudinal ligament. Photomed Laser Surg. 2008;26(2):119-127.

10. Iwasaki M, Okuda S, Miyauchi A, et al. Surgical strategy for cervical myelopathy due to ossification of the posterior longitudinal ligament: part 2: advantages of anterior decompression and fusion over laminoplasty. Spine (Phila Pa 1976). 2007;32(6):654-660.

11. Masaki Y, Yamazaki M, Okawa A, et al. An analysis of factors causing poor surgical outcome in patients with cervical myelopathy due to ossification of the posterior longitudinal ligament: anterior decompression with spinal fusion versus laminoplasty. J Spinal Disord Tech. 2007;20(1):7-13.

12. Tani T, Ushida T, Ishida K, Iai H, Noguchi T, Yamamoto H. Relative safety of anterior microsurgical decompression versus laminoplasty for cervical myelopathy with a massive ossified posterior longitudinal ligament. Spine (Phila Pa 1976). 2002;27(22):2491-2498.

13. Xu J, Zhang K, Ma X, et al. Systematic review of cohort studies comparing surgical treatment for multilevel ossification of posterior longitudinal ligament: anterior vs posterior approach. Orthopedics. 2011;34(8):e397-e402.

14. Luo J, Cao K, Huang S, et al. Comparison of anterior approach versus posterior approach for the treatment of multilevel cervical spondylotic myelopathy. Eur Spine J. 2015;24(8):1621-1630.

15. Zhu B, Xu Y, Liu X, Liu Z, Dang G. Anterior approach versus posterior approach for the treatment of multilevel cervical spondylotic myelopathy: a systemic review and meta-analysis. Eur Spine J. 2013;22(7):1583-1593.

16. Liu X, Min S, Zhang H, Zhou Z, Wang H, Jin A. Anterior corpectomy versus posterior laminoplasty for multilevel cervical myelopathy: a systematic review and meta-analysis. Eur Spine J. 2014;23(2):362-372.

17. Liu X, Wang H, Zhou Z, Jin A. Anterior decompression and fusion versus posterior laminoplasty for multilevel cervical compressive myelopathy. Orthopedics. 2014;37(2):e117-e122.

18. Sun Y, Li L, Zhao J, Gu R. Comparison between anterior approaches and posterior approaches for the treatment of multilevel cervical spondylotic myelopathy: a meta-analysis. Clin Neurol Neurosurg. 2015; 134:28-36.

19. Yamazaki A, Homma T, Uchiyama S, Katsumi Y, Okumura H. Morphologic limitations of posterior decompression by midsagittal splitting method for myelopathy caused by ossification of the posterior longitudinal ligament in the cervical spine. Spine (Phila Pa 1976). 1999;24(1):32-34.

20. Fujiyoshi T, Yamazaki M, Kawabe J, et al. A new concept for making decisions regarding the surgical approach for cervical ossification of the posterior longitudinal ligament: the $K$-line. Spine (Phila Pa 1976). 2008;33(26):E990-E993.

21. Nishida N, Kanchiku T, Kato Y, et al. Biomechanical analysis of cervical myelopathy due to ossification of the posterior longitudinal ligament: effects of posterior decompression and kyphosis following decompression. Exp Ther Med. 2014;7(5):1095-1099.

22. Ogawa $Y$, Chiba K, Matsumoto M, et al. Long-term results after expansive open-door laminoplasty for the segmental-type of ossification of the posterior longitudinal ligament of the cervical spine: a comparison with nonsegmental-type lesions. J Neurosurg Spine. 2005;3(3):198-204.

23. Goto S, Kita T. Long-term follow-up evaluation of surgery for ossification of the posterior longitudinal ligament. Spine (Phila Pa 1976). 1995;20(20):2247-2256.

24. Goel A, Nadkarni T, Shah A, Rai S, Rangarajan V, Kulkarni A. Is only stabilization the ideal treatment for ossified posterior longitudinal ligament? Report of early results with a preliminary experience in 14 patients. World Neurosurg. 2015;84(3):813-819. 
25. Yuan W, Zhu Y, Liu X, et al. Postoperative three-dimensional cervical range of motion and neurologicalal outcomes in patients with cervical ossification of the posterior longitudinal ligament: cervical laminoplasty versus laminectomy with fusion. Clin Neurol Neurosurg. 2015; 134:17-23.

26. Lee CH, Jahng TA, Hyun SJ, Kim KJ, Kim HJ. Expansive laminoplasty versus laminectomy alone versus laminectomy and fusion for cervical ossification of the posterior longitudinal ligament: is there a difference in the clinical outcome and sagittal alignment? J Spinal Disord Tech. Epub July 29, 2014.

27. Iwasaki M, Kawaguchi Y, Kimura T, Yonenobu K. Long-term results of expansive laminoplasty for ossification of the posterior longitudinal ligament of the cervical spine: more than 10 years follow up. J Neurosurg. 2002;96(2 Suppl):180-189.

28. Matsuoka T, Yamaura I, Kurosa Y, Nakai O, Shindo S, Shinomiya K. Long-term results of the anterior floating method for cervical myelopathy caused by ossification of the posterior longitudinal ligament. Spine (Phila Pa 1976). 2001;26(3):241-248.

29. Yang H, Lu X, Wang X, et al. A new method to determine whether ossified posterior longitudinal ligament can be resected completely and safely: spinal canal "Rule of Nine" on axial computed tomography. Eur Spine J. 2015;24(8):1673-1680.
30. Wang X, Chen D, Yuan W, Zhang Y, Xiao J, Zhao J. Anterior surgery in selective patients with massive ossification of posterior longitudinal ligament of cervical spine: technical note. Eur Spine J. 2012;21(2): 314-321.

31. Singh K, Vaccaro AR, Kim J, Lorenz EP, Lim TH, An HS. Biomechanical comparison of cervical spine reconstructive techniques after a multilevel corpectomy of the cervical spine. Spine (Phila Pa 1976). 2003;28(20):2352-2358.

32. Vaccaro AR, Falatyn SP, Scuderi GJ, et al. Early failure of long segment anterior cervical plate fixation. J Spinal Disord. 1998;11(5):410-415.

33. Acosta FL Jr, Aryan HE, Chou D, Ames CP. Long-term biomechanical stability and clinical improvement after extended multilevel corpectomy and circumferential reconstruction of the cervical spine using titanium mesh cages. J Spinal Disord Tech. 2008;21(3):165-174.

34. Hussain M, Nassr A, Natarajan RN, An HS, Andersson GB. Biomechanical effects of anterior, posterior, and combined anterior-posterior instrumentation techniques on the stability of a multilevel cervical corpectomy construct: a finite element model analysis. Spine J. 2011; 11(4):324-330.
Therapeutics and Clinical Risk Management

\section{Publish your work in this journal}

Therapeutics and Clinical Risk Management is an international, peerreviewed journal of clinical therapeutics and risk management, focusing on concise rapid reporting of clinical studies in all therapeutic areas outcomes, safety, and programs for the effective, safe, and sustained use of medicines. This journal is indexed on PubMed Central, CAS,

\section{Dovepress}

EMBase, Scopus and the Elsevier Bibliographic databases. The manuscript management system is completely online and includes a very quick and fair peer-review system, which is all easy to use. Visit http://www.dovepress.com/testimonials.php to read real quotes from published authors.

Submit your manuscript here: http://www.dovepress.com/therapeutics-and-clinical-risk-management-journal 